\title{
A Quality Improvement Project to Minimize COVID-19 Infections in Patients Receiving Haemodialysis and the Role of Routine Surveillance Using Nose and Throat Swabs for SARS-CoV-2 rRT-PCR and Serum Antibody Testing
}

\author{
Dimitrios Poulikakos $^{a, b}$ Rajkumar Chinnadurai ${ }^{a, b} \quad$ Yvonne Mcgee $^{a}$ \\ Simon Gray ${ }^{a}$ Toni Clough ${ }^{a}$ Nicola Clarke ${ }^{a}$ Tracey Murphy ${ }^{a}$ Olivia Wickens ${ }^{a}$ \\ Carol Mitchellc Denise Darby ${ }^{d}$ Joel Paule Paul Chadwick ${ }^{f}$ Su Sethi ${ }^{g}$ \\ Smeeta Sinha, ${ }^{a}$ Philip A. Kalra ${ }^{a}$ b Rosie Donne ${ }^{a, b}$ \\ aRenal Department, Salford Royal NHS Foundation Trust, The Northern Care Alliance NHS Group, Manchester, UK; \\ ${ }^{b}$ Faculty of Biology, Medicine and Health, University of Manchester, Manchester, UK; Infection Control Department, \\ Salford Royal NHS Foundation Trust, The Northern Care Alliance NHS Group, Manchester, UK; ${ }^{d}$ Chemical Pathology \\ Department, Salford Royal NHS Foundation Trust, The Northern Care Alliance NHS Group, Manchester, UK; 'Virology \\ Department, The Northern Care Alliance NHS Group, Manchester, UK; ${ }^{f}$ Microbiology Department, Salford Royal \\ NHS Foundation Trust, The Northern Care Alliance NHS Group, Manchester, UK; 9Public Health England, North West \\ Region Specialised Commissioning Team, NHS, London, UK
}

\section{Keywords}

Antibodies · COVID-19 · Haemodialysis · Infection prevention control measures · Quality improvement . SARS-CoV-2

\begin{abstract}
Background: Patients receiving in-centre haemodialysis (ICHD) are highly vulnerable to COVID-19. Objective: We created a quality improvement (QI) project aimed to eliminate outbreaks of COVID-19 in haemodialysis units and evaluated the utility of surveillance rRT-PCR test and SARS-CoV-2 serum antibodies for prompt identification of patients infected with COVID-19. Methods: A multifaceted QI programme including a bundle of infection prevention control (IPC) mea-
\end{abstract}

karger@karger.com www.karger.com/nef

Karger $\frac{1}{\%}$
(C) 2021 The Author(s)

Published by S. Karger AG, Basel

This is an Open Access article licensed under the Creative Commons Attribution-NonCommercial-4.0 International License (CC BY-NC) (http://www.karger.com/Services/OpenAccessLicense), applicable to the online version of the article only. Usage and distribution for commercial purposes requires written permission. sures was implemented across 5 ICHD units following the first wave of the pandemic in June 2020. Primary outcomes evaluated before and after QI implementation were incidence of outbreaks and severe COVID-19 illness defined as COVID-19-related death or hospitalization. Secondary outcomes included the proportion of patients identified in the pre-symptomatic/asymptomatic phase on surveillance rRTPCR screening and the incidence and longevity of SARSCoV-2 antibody response. Results: Following the implementation of the QI project, there were no further outbreaks. Preand post-implementation comparison showed a significant reduction in COVID-19-related mortality and hospitalization (26 vs. 13 events, respectively, $p<0.001$ ). Surveillance rRTPCR screening identified 39 asymptomatic or pre-symptomatic cases out of a total of 59 rRT-PCR-positive patients 
(39/59, 66\%). SARS-CoV-2 antibody levels were detected in 72/74 (97\%) rRT-PCR-positive patients. Amongst rRT-PCRpositive patients diagnosed before August 2020, 96\% had detectable antibodies until January 2021 (days from the rRTPCR test to last antibody testing, 245-280). Conclusions: Systematic implementation of a bundle of IPC measures using QI methodology and surveillance rRT-PCR eliminated outbreaks in HD facilities. Most HD patients mount and sustain antibody response to COVID-19 for over 8 months.

(c) 2021 The Author(s).

Published by S. Karger AG, Basel

\section{Introduction}

Patients receiving in-centre haemodialysis (ICHD) are at high risk from COVID-19 due to existing comorbidities with short-term mortality exceeding 20\% [1]. Most importantly, ICHD patients are at high risk for acquiring COVID-19 infection because they are unable to self-isolate having to visit haemodialysis (HD) units 3 times per week for life-maintaining treatment [1]. By August 2020, $11.3 \%$ (2339) of ICHD patients in England had contracted COVID-19 [2], and by November 2020, 662 patients, approximately $3 \%$ of all ICHD patients in the United Kingdom (UK), had succumbed to COVID-19 [3].

Guidelines and recommendations were issued rapidly by the renal community [4-6] aiming at protecting this vulnerable population. Infection prevention control (IPC) recommendations focused on minimizing the risk of COVID-19 transmission in dialysis units applying screening and triage processes, face masking, physical distancing measures, and isolation protocols to ensure care is maintained with minimal cross-infection risk. The ensuing challenge was to design, introduce, and maintain new unprecedented and demanding patterns of work adjusted to the pandemic IPC strategy.

During the first wave (April-July 2020), diagnostic testing with nose and throat SARS-CoV-2 real-time reverse transcription polymerase chain reaction (rRT-PCR) swabs was limited to suspected or confirmed cases due to both limited capacity and the assumption that symptomatic individuals were the only drivers of transmission. Subsequent studies reported high viral loads in asymptomatic and pre-symptomatic patients infected with SARS-CoV-2 [7], highlighting the need for early detection and prompt isolation of pre-symptomatic or asymptomatic individuals to prevent nosocomial infections. Consequently, it was plausible that integration of regular surveillance with RT-PCR in asymptomatic ICHD patients was likely to have a role in reducing transmission.
In addition, regular SARS-CoV-2 antibody sero-surveillance in this population might have complemented monitoring of effectiveness of IPC screening programmes in the dialysis units and provide information about the duration of detectable SARS-CoV-2 antibodies in ICHD patients.

The COVID-19 pandemic has required an extraordinarily rapid change and adjustment of health care services based on challenges and evolving knowledge about COVID-19. To this end, we established a quality improvement project aiming to minimize COVID-19 transmission in the HD units by using rapid learning cycles to implement and maintain nationally recommended IPC protocols. We also integrated surveillance nose and throat rRT-PCR and antibody testing in order to optimize the efficacy of screening and surveillance programme.

\section{Methods}

\section{Setting}

The Renal Unit

The Salford renal unit is one of the 52 renal units in England and provides renal services for Greater Manchester North with a catchment population area of approximately 1.55 million and a population receiving renal replacement therapy that has been ranked as the 7th most socially deprived amongst the 52 units based on the Index of Multiple Deprivation (online suppl. File 1; see www.karger.com/doi/10.1159/000520654 for all online suppl. material). There were 402 patients on ICHD receiving treatment in 1 main and 4 satellite HD units in March 2020. By August 12, the number of deaths associated with COVID-19 in the UK had reached 46,706, and our region (North West of England) had the second highest rate of cases after London [8]. Baseline demographics of the HD population are shown in Table 1.

National IPC and Public Health Interventions

The IPC guidance from Public Health England issued on March 3, 2020, was followed [9]. Universal surgical masks for ICHD patients and nursing staff in HD units were introduced following UK Renal Association recommendations on April 5, 2020 [10].

Dialysis patients were advised to self-isolate and were covered by the national shielding programme that included financial support for self-isolation from March 2020 until August 1 when the programme was paused for all extremely vulnerable individuals in England [11]. The shielding programme was re-introduced on December 20, 2020, in areas with high COVID-19 community rates (tier 4). The first strict national lockdown started on March 23, 2020, until June 1, 2020, when restrictions were eased with further easing on July 31 . The second national lockdown started on November 5, 2020, with closing of non-essential services and businesses including hospitality while essential shops and educational settings remained open until January 6, 2021, when schools were closed. The timeline of public health interventions is shown in Figure 1. 
Table 1. Baseline characteristics of the prevalent haemodialysis patients

\begin{tabular}{ll}
\hline Variables & $N, 402$ \\
\hline Age, years & $62(51-73)$ \\
Gender, male & $261(65)$ \\
Ethnicity, Caucasian & $277(68.9)$ \\
Body mass index, kg/m ${ }^{2}$ & $26.7(23.2-30.9)$ \\
Primary renal disease & \\
Diabetic nephropathy & $132(32.8)$ \\
Hypertensive/renovascular disease & $57(14.2)$ \\
Glomerulonephritis & $52(12.9)$ \\
ADPKD & $28(6.9)$ \\
Urological & $37(9.2)$ \\
Pyelonephritis & $14(3.5)$ \\
ANCA-associated vasculitis & $9(2.23)$ \\
Others & $33(8.2)$ \\
Unknown & $40(9.9)$ \\
Comorbidity & \\
Ischaemic heart disease & $65(16.2)$ \\
Heart failure & $23(5.7)$ \\
Cerebrovascular accident & $41(10.1)$ \\
Diabetes mellitus & $163(40.5)$ \\
Angiotensin-converting enzyme inhibitor use & $76(18.9)$ \\
Angiotensin II receptor blocker use & $51(12.7)$ \\
\hline
\end{tabular}

Continuous variables are expressed as median and interquartile range. Categorical variables are expressed as $n$ and percentage. ADPKD, autosomal dominant polycystic kidney disease; ANCA, antinuclear cytoplasmic antibody.

Local Practice and Interventions prior to the QI Project

Prior to the peak of the pandemic in North West of England (March 23, 2020), 166 suitable patients from our ICHD population were switched temporarily to twice-weekly HD in their respective dialysis units in order to reduce exposure risk to COVID-19 [12], and the main unit at Salford Royal Hospital was designated as a COVID-19 treatment area. A screening process was implemented to identify those patients with possible COVID-19 symptoms or close contacts of a symptomatic person before they attended for dialysis. This included advising patients to report symptoms before travelling to their dialysis session and a triage system on entry to the dialysis unit entailing temperature check and a short symptom questionnaire. Patients with suspected or confirmed COVID-19 or with a household contact with cases were directed to the designated COVID-19 unit for HD treatment. Patients with close ICHD contact were screened with nose and throat swabs at their unit.

IPC policies were introduced as per national guidance including universal masking, hand hygiene, physical distancing measures during transportation and waiting areas, environmental cleaning, and disinfection. During the first wave (March 19-June $26,2020)$, the whole main unit was dedicated for COVID-19 isolation purposes. In June 2020, the main unit was divided with solid doors into a COVID-19 fully segregated COVID-19 zone and a COVID-19-free area. The vast majority of ICHD patients receiving twice-weekly HD treatment reverted back to thrice-weekly treatment. On July 1, 2020, our centre had the lowest COVID-19 HD unit death rate of $1.5 \%$ in the region, almost $30 \%$ lower than the regional data $(2.2 \%)$, with the case fatality rate being $14.6 \%$ compared to $25.8 \%$ for the whole of England [13].

\section{Contact and Outbreak Definitions}

Close ICHD contact was defined as a patient who dialysed on the same shift, shared transport, or shared waiting room for $>15$ min. Household contact was defined as a patient sharing the same household with a person diagnosed with COVID-19 infection. An outbreak was defined as 2 or more patients with positive COVID-19 rRT-PCR result within 14 days who were linked in time and place.

\section{Baseline Assessment}

An outbreak of COVID-19 occurred in one of the satellite units (A) during the first week of April 2020 (i.e., prior to the introduction of universal masking). Surveillance rRT-PCR screening was conducted in all patients deemed as close contacts. Overall, 15 patients tested positive of whom 8 required inpatient care and 4 died. A structured investigation to inform the response was conducted (online suppl. File 2). A second cluster of 4 cases was identified in another satellite unit (B) shortly after, including a case who moved from satellite unit A to satellite unit B several days before developing symptoms of COVID-19. The QI project was initiated in response to this outbreak, aiming to minimize the risk of transmission of COVID-19 in the dialysis environment. This included the need for constant evaluation and improvement of IPC measures and regular engagement of all stakeholders to ensure IPC practices were embedded and maintained.

\section{QI Project Set-Up}

The QI project started on June 9, 2020, with the formation of a multidisciplinary team comprising the nurse manager from each dialysis unit, senior renal nurses, infection control nurse, QI lead, digital program director, renal consultants with an interest in HD, and senior administrative staff. Some but not all staff had experience of leading or participating in QI work. The Institute for Healthcare Improvement "Model for Improvement" framework was used to set an aim, define measures, and use Plan, Do, Study, and Act (PDSA) cycles to test and improve change [14]. The project aim was to prevent COVID-19 transmission in dialysis units. Remote meetings were held weekly for the first 6 weeks and then fortnightly thereafter to maintain a shared purpose to protect patients and staff from COVID-19 infection across all 5 dialysis units. The 10 key IPC precautions are summarized in Figure 2, and the main QI project interventions are shown as a driver diagram in online supplementary File 3.

Implementation

A Gantt chart summarizing project activity from May to December 2020 is shown in Figure 1. The key PDSA cycles of the project are summarized below.

PDSA 1 - Electronic Report of Dialysis Patients with COVID19-Positive PCR Results

A mandatory field was created on the electronic patient record (EPR) haemodialysis document to record COVID-19 symptoms, dialysis unit and dialysis station number, timing of dialysis, and travel details. This was linked to an electronic report showing all 


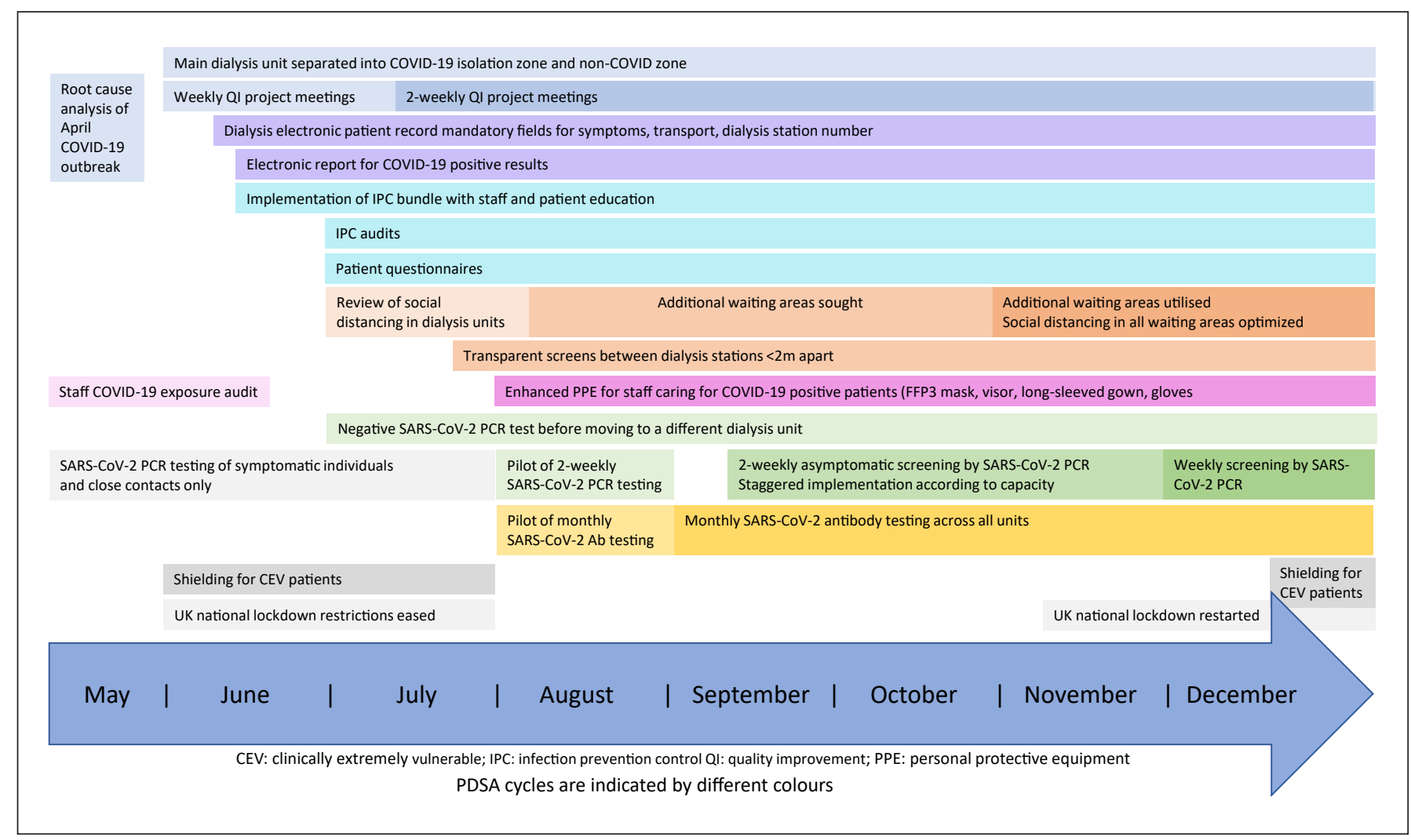

Fig. 1. Gantt chart of quality improvement interventions and public health measures. PDSA cycles are indicated by different colours. $\mathrm{CEV}$, clinically extremely vulnerable; IPC, infection prevention control; QI, quality improvement; PPE, personal protective equipment.

Fig. 2. The 10 key IPC precautions. IPC, infection prevention control.

$\begin{array}{ll}\text { I } & \text { Patient education on COVID-19 symptoms and IPC precautions } \\ \text { II } & \text { Staff education on COVID-19 symptoms, IPC precautions and working practices } \\ \text { III } & \text { Environmental cleaning } \\ \text { IV } & \text { Hand hygiene at key points } \\ \text { V } & \text { Social distancing of }>2 \text { m during travel, waiting areas and dialysis } \\ \text { VI } & \text { PPE for patients (mask), drivers and nurses } \\ \text { VII } & \text { Symptoms screening by driver and dialysis staff } \\ \text { VIII } & \text { Prompt COVID-19 PCR testing for suspected infection } \\ \text { IX } & \text { Optimal isolation/cohorting/de-isolation } \\ \text { X } & \text { Contact tracing of patients with positive PCR tests }\end{array}$

HD patients with positive COVID-19 PCR results. An email alert system was implemented for new COVID-19-positive PCR results to dialysis unit managers and senior staff. This facilitated rapid alerting of patients requiring transfer to the COVID-19 isolation unit and contact tracing of individuals at risk. It also highlighted clustering of cases within the same dialysis unit requiring further scrutiny for possible transmission within the dialysis unit.

\section{PDSA 2 - IPC Precautions Bundle}

A bundle of 10 key IPC precautions was implemented across all dialysis units, assisted by education for staff and patients
(Fig. 2). Patients were advised to report by telephone to the unit if they had symptoms in keeping with COVID-19 or close contact with a suspected or confirmed case. For patients requiring hospital transport, a symptom questionnaire was utilized by the driver as shown in the Quick Reference Guide (online suppl. File 4). Further screening was performed prior to entering the HD unit and symptoms recorded in the EPR document. IPC audits were performed weekly for each dialysis unit to highlight any areas requiring improvement. A 10-point patient questionnaire (online supplementary File 5) was developed as a process measure of reliable implementation of the above IPC precautions. Ten patients (10- 
$15 \%)$ from each dialysis unit were sampled weekly, which highlighted areas for improvement or retraining. After 2 months, this was reduced in frequency to monthly as results were consistently good.

\section{PDSA 3 - Social Distancing between Dialysis Stations and in} Waiting Areas

All dialysis unit managers reviewed social distancing between dialysis stations and in waiting areas to identify areas where a 2-m distance between patients could not be maintained. Transparent acrylic screens were successfully implemented in some areas to separate dialysis stations. In some areas, they proved unsafe due to inadequate space for emergency care if needed. Transparent curtains were then implemented in these problematic areas, with satisfactory feedback from staff and patients. Waiting areas were reconfigured to allow 1-way patient flow and maximize social distancing. Neighbouring clinic rooms were adapted to increase waiting area capacity. Other areas of the building were utilized as waiting areas, which allowed separation of patients arriving from those awaiting transport home after dialysis. This reduced the number of possible close contacts for any individual patient attending for dialysis, thereby reducing the chance of transmission of infection.

\section{PDSA 4 - Enhanced PPE for COVID-19 Isolation Area}

An occupational risk exposure audit was conducted at the end of the first wave of the pandemic showing increased risk of COVID-19 infection in HD nurses covering the COVID-19 isolation area [15] with $23 \%$ having been diagnosed with COVID-19 on nose and throat swabs. Based on this finding, we implemented enhanced PPE precautions using visors, filtering facepiece 3 (FFP3) mask, and long sleeved fluid-repellent surgical gown and gloves for nurses caring for suspected or confirmed COVID-19 patients. This was found to be acceptable to staff, who were reassured to wear the maximal protection. All staff were face-fit tested for the available FFP3 masks and most were able to achieve a good fit. Some staff did not pass a face-fit test and did not care for COVID-19-positive patients. The supply of masks changed during the pandemic requiring repeat fit testing. A member of the staff was trained to become a fit-tester and maintained a database of staff and appropriate FFP3 masks for use.

PDSA 5 - Surveillance Screening with Nose and Throat SARSCoV-2 rRT-PCR Test and Antibody Testing

Guidance was developed mandating a patient having a negative COVID-19 swab within the $72 \mathrm{~h}$ before commencing haemodialysis in an outpatient satellite dialysis unit. This included all patients repatriated to the non-isolation area of the main unit in June 2020 following the change in dialysis frequency to thrice weekly.

Surveillance nose and throat swab SARS-CoV-2 rRT-PCR testing in asymptomatic HD patients was piloted in August 2020 in 2 satellite units with rising community rates. The surveillance programme was paused from August 20, 2020, to September 7, 2020, due to laboratory testing capacity pressures. From September 2020, fortnightly surveillance nose and throat rRT-PCR screening was implemented across all units, and the frequency increased to weekly intervals in December 2020 when the laboratory testing capacity increased. Monthly routine sero-surveillance with SARSCoV-2 antibody testing was started in August 2020 in all HD patients.

Minimizing COVID-19 Infection in

Haemodialysis Units

\section{SARS-CoV-2 rRT-PCR Test}

A total of 4 different nucleic acid amplification tests (PCR) were used across our 2 laboratories using the methodology described in the kit inserts after local verification. The gene targets used in the different assays are listed in online supplementary File 6.

\section{SARS-CoV-2 Antibody Testing}

All sera collected were tested for COVID-19 IgG antibody with the Siemens' immunoassay using acridinium ester chemiluminescent technology detecting antibodies targeting the spike protein S1 RBD [16]. An index $\geq 1.0$ was deemed positive according to the cutoff assigned by the manufacturer. Sensitivity and specificity for the assay were reported at $98.1 \%$ and $99.9 \%$, respectively. The Siemens' immunoassay became available in the hospital laboratory from 12 June 2020.

\section{Project Measures}

Outcome measures were the number of new cases of COVID-19 per week in each dialysis unit and number of outbreaks. Pre- and post-QI project implementation comparison of COVID-19 deaths and hospitalizations was conducted prior to the roll out of COVID-19 vaccination in ICHD patients. Process measures were COVID-19 infection rates, weekly infection control audits, and patient feedback questionnaires.

Analysis was also performed to identify (a) the number of asymptomatic or pre-symptomatic patients detected on surveillance rRT-PCR screening, (b) the percentage of rRT-PCR-positive HD patients who develop detectable SARS-CoV-2 antibodies, and (c) the duration of detectable antibodies in rRT-PCR-positive and rRT-PCR-negative but SARS-CoV-2 antibody-positive patients identified from March to August 2020. Balancing measures were the unintended consequences of the IPC measures, reported by staff during QI meetings and by patients via the patient questionnaires.

\section{Statistical Analysis}

The $\chi^{2}$ test was used for categorical values and the Mann-Whitney $\mathrm{U}$ test for non-normally distributed continuous values. A $p$ value $<0.05$ was considered statistically significant in this study. SPSS version-23 licenced to the University of Manchester was used for statistical analysis.

\section{Data Collection and Study Registration}

Data were collected from the EPR and from the electronic report designed for the QI project. The study was registered with the Northern Care Alliance Research and Innovation Department (Ref. No. S21HIP08). As this is a quality improvement project with complete anonymization of patient identification details, the need for individual consenting was waived.

\section{Results}

Between April and December 2020, a total of 102 patients had a positive SARS-CoV-2 rRT-PCR test of which $56(54.9 \%)$ were asymptomatic when tested. In this period, a total of 11 deaths and 28 hospitalizations were reported due to COVID-19. 
Table 2. COVID-19-positive cases and associated outcomes (April-December 2020)

\begin{tabular}{llll}
\hline & April-July 2020 & August-December 2020 & Total \\
\hline Total rRT-PCR-positive patients & 43 & 59 & 102 \\
Asymptomatic on diagnosis, $n(\%)$ & $17 / 43(39.5)$ & $39 / 59(66.1)$ & $56 / 102(54.9)$ \\
Detectable SARS-CoV-2 antibodies in rRT-PCR-positive cases, $n(\%)$ & $26 / 28(92.8)$ & $46 / 46(100)$ & $72 / 74(97)$ \\
rRT-PCR-negative SARS-CoV-2 antibody-positive patients & 12 & 0 & 12 \\
Outcomes & & 5 & 11 \\
$\quad$ COVID-19 deaths & 6 & 8 & 28 \\
$\quad$ COVID-19 hospitalizations & 20 & $13 / 59(22.1)$ & 39 \\
Combined outcome & $26 / 43(60.5)$ & & $p$ value \\
\hline
\end{tabular}

Table 3. Trend of SARS-CoV-2 lgG antibody status in patients from the first wave (April-July)

\begin{tabular}{|c|c|c|c|c|c|c|}
\hline Variable & First (Aug) & Second (Sep) & Third (Oct) & Fourth (Nov) & Fifth (Dec) & Sixth (Jan) \\
\hline \multicolumn{7}{|l|}{ COVID-19 rRT-PCR-positive, $N=43$} \\
\hline SARS-CoV-2 IgG antibody positive, $n(\%)$ & $26 / 28(92.8)$ & $24 / 25(96)$ & $26 / 28(92.8)$ & $22 / 24(91.6)$ & $29 / 29(100)$ & $24 / 25(96)$ \\
\hline \multicolumn{7}{|l|}{ COVID-19 rRT-PCR negative, $N=12$} \\
\hline SARS-CoV-2 IgG antibody positive, $n(\%)$ & $12 / 12(100)$ & $8 / 8(100)$ & $7 / 7(100)$ & $10 / 10(100)$ & $11 / 11(100)$ & $4 / 4(100)$ \\
\hline
\end{tabular}

Categorical variables are expressed as number (\%). Continuous variables are expressed as median (interquartile range).

\section{Primary Outcomes}

Since the implementation of the QI project (June-July 2020) until December 2020, there were no further COVID-19 outbreaks in the dialysis units. During this same period, the composite outcome of deaths and hospitalizations due to COVID-19 decreased to 13 events in 59 rRTPCR-positive patients compared to 26 events in $43 \mathrm{rRT}$ PCR-positive patients prior to the initiation of the QI project $(p<0.001)$ (Table 2).

\section{Secondary Outcomes}

There were 17 out of a total of 43 (39\%) and 39 out of a total of 59 (66\%) rRT-PCR-positive cases diagnosed in the asymptomatic or pre-symptomatic phase before and after the implementation of routine rRT-PCR surveillance, respectively. Out of a total of 102 rRT-PCR-positive cases from the beginning of the pandemic, 74 underwent antibody testing and 72 (97\%) had detectable levels. There were $28 \mathrm{rRT}$-PCR-positive patients diagnosed with COVID-19 on rRT-PCR prior to August 2020 with available antibody status and 1 more patient had an antibody test in December. Amongst the 25 patients who had follow-up antibody testing in January 2021, 24 (96\%) had detectable antibodies over a median duration of 272 days. Twelve rRT-PCR-negative patients were SARS-CoV-2 antibody positive in August 2020, and antibodies remained detectable on follow-up although the number of available samples decreased over time. Table 3 illustrates the monthly antibody status of the rRT-PCR-positive and -negative patients from the first wave (April-July). Monthly new rRT-PCR and antibody cases are presented in Figure 3.

There were 45 SARS-CoV-2 antibody tests performed in December 2020 that were excluded from the analysis due to false reactive results observed with certain reagent lots. Few patients had antibody tests prior to August 2020 requested on clinical grounds.

Unintended consequences identified during the project were patient discomfort while wearing masks, increased time taken for cleaning, required increase in staffing, and requirement for additional waiting area space. No patients refused nose and throat swabs. 
Fig. 3. Monthly distribution of new COVID rRT-PCR-positive and new SARSCoV-2 antibody-positive cases.

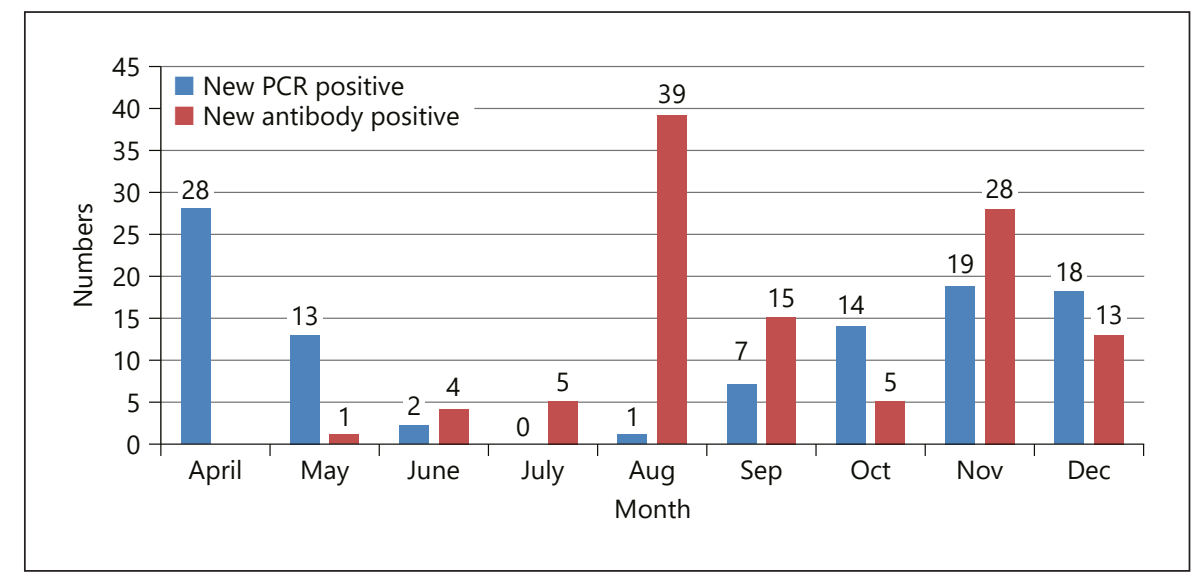

\section{Discussion}

This quality improvement project showed that implementation of an IPC bundle can eliminate COVID-19 outbreaks in dialysis units and reduce the incidence of COVID-19-related deaths and hospitalizations. Surveillance rRT-PCR played a central role in early identification and prompt isolation of infected patients. In our cohort, the vast majority of ICHD diagnosed with COVID-19 on rRT-PCR developed SARS-CoV-2 antibodies, and most of the patients diagnosed during the first wave sustained detectable levels for $>8$ months.

The quality improvement methodology enabled multidisciplinary engagement for a rapid and extensive operational change [17]. Live data collection and continuous evaluation of the various interventions within the QI framework was extremely important during a period with scarce evidence-based tools and lack of randomized controlled studies for most of the IPC tools in dialysis units.

Nineteen out of 43 (44\%) rRT-PCR-confirmed cases during the first wave occurred in the context of outbreaks prior to the introduction of universal masking and testing, highlighting the importance of masks and testing for this vulnerable population. The reduction of deaths and hospitalizations during the second wave can be attributed to reduced viral loads due to universal masking [18] and enhanced nation-wide IPC measures, better understanding of management of COVID-19 infection, and improved rRT-PCR testing capacity allowing early identification and isolation of cases. Across the North West region, the cumulative ICHD mortality rate decreased from $2.8 \%$ on 29 July [19] to $2.0 \%$ on January 17,2021 [20], with a parallel increase of cumulative rRT-PCR de- tected cases from $8.9 \%$ to $13 \%$, respectively. During the same periods in our ICHD population, we recorded a decrease in the mortality rate from $1.8 \%$ [19] to $1.3 \%$ [20], the lowest mortality rate in our region, indicating effective IPC implementation using a QI approach and the introduction of surveillance rRT-PCR screening. Our results showing high rates and duration of detectable SARS-CoV-2 antibodies are in keeping with a recent study in ICHD patients [21] showing sustained detectable immune response for the vast majority sustained for 6 months after SARS-CoV-2 infection including patients who had mild or asymptomatic infection.

Limitations of this study include the lack of quantitative (viral load estimation) rRT-PCR and of quantitative SARS-CoV-2 antibody testing. In addition, the variation of population prevalence of SARS-CoV-2 throughout the outbreak, particularly in the first wave where testing was limited and focussed on symptomatic patients, limited our conclusions. Due to the nature of the project, we were unable to analyse the relative contribution of the various public health interventions during the duration of the project. The ICHD population changed during the process due to new incident patients, change in modality (home HD or peritoneal dialysis), kidney transplantation, or death. Surveillance antibody testing was performed with routine monthly bloods and not as part of a research protocol, and as a result, some patients occasionally missed antibody testing due to temporary alterations of dialysis days or shifts.

Our study shows that the QI methodology is a valuable tool for rapid changes during crisis management and evaluation of novel interventions aiming at patient safety. Surveillance rRT-PCR has a role in reducing COVID-19 transmission in HD units. 


\section{Acknowledgments}

The authors thank all the members of the Quality Improvement Project, the biochemistry, microbiology, and virology departments, all of the HD unit staff who have supported this project, and the Salford Royal NHS Foundation Trust Senior Teams for their support of this project.

\section{Statement of Ethics}

This project has been performed in accordance with the Declaration of Helsinki. The study was registered with the Northern Care Alliance Research and Innovation Department (Ref. No. S21HIP08). As this is a quality improvement project with complete anonymization of patient identification details, the need for individual consenting was waived.

\section{Conflict of Interest Statement}

The authors declare no conflicts of interest.

\section{Funding Sources}

The authors received no specific funding for this work.

\section{Author Contributions}

All the authors contributed equally to this work and approved the version to be published.

\section{Data Availability Statement}

The datasets used and analysed during the current study are available from the corresponding author on reasonable request.

\section{References}

1 Hsu CM, Weiner DE. COVID-19 in dialysis patients: outlasting and outsmarting a pandemic. Kidney Int. 2020 Dec;98(6):1402-4.

2 COVID-19 surveillance report for renal centres in the UK. A summary of the first wave of the pandemic - March to August 2020: UK Renal Registry; 2021. Available from: https:// renal.org/sites/renal.org/files/covid_report_ first_wave_FINAL_041220.pdf.

3 COVID-19 vaccination for adult patients with kidney disease: a position statement from the UK renal community, joint renal societies (British Renal Society, British Transplantation Society, Renal Association) and charity partners at the National Kidney Federation and Kidney Care UK statement. https: //renal.org/health-professionals/covid-19/raresources/covid-19-vaccination- adult-patients-kidney-disease.

4 Basile C, Combe C, Pizzarelli F, Covic A, Davenport A, Kanbay M, et al. Recommendations for the prevention, mitigation and containment of the emerging SARS-CoV-2 (COVID-19) pandemic in haemodialysis centres. Nephrol Dial Transplant. 2020 May 1;35(5):737-41.

5 Kliger AS, Silberzweig J. Mitigating risk of COVID-19 in dialysis facilities. Clin J Am Soc Nephrol. 2020 May 7;15(5):707-9.

6 Recommendations for minimising the risk of transmission of COVID-19 in UK adult haemodialysis units. KQuIP COVID-19 HD Ensuring Patient Safety Work Stream published June 26, 2020. https://britishrenal.org/wpcontent/uploads/2020/07/Recommendations-for-minimising-risk-of-transmissionof-COVID-19-on-UK-ha._-002.pdf.

7 Cevik M, Tate M, Lloyd O, Maraolo AE, Schafers J, Ho A. SARS-CoV-2, SARS-CoV, and MERS-CoV viral load dynamics, duration of viral shedding, and infectiousness: a systematic review and meta-analysis. Lancet $\mathrm{Mi}$ crobe. 2021 Jan;2(1):e13-22.

8 COVID-19: The view from the North West, Public Health Matters, Public Health England. https://publichealthmatters.blog.gov. uk/2021/03/01/covid-19-the-view-from-thenorth-west/.

9 Coronavirus (COVID-19): guidance, published 3 March 2020, Public Health England. https://www.gov.uk/government/collections/ coronavirus-covid-19-list-of-guidance.

$10 \mathrm{PPE}$ and use of masks by dialysis patients. The British Renal Society website. https://britishrenal.org/ppe-and-use-of-masks-by-dialysis-patients/.

11 British Renal Society/Renal Association group to assess and explain stratified risk of COVID-19 (coronavirus) in kidney patients. https: //britishrenal.org/news/british-renal-societyrenal-association-group-to-assess-and-explain-stratified-risk-of-covid-19-coronavirusin-kidney-patients/.

12 Lodge MDS, Abeygunaratne T, Alderson $\mathrm{H}$, Ali I, Brown N, Chrysochou C, et al. Safely reducing haemodialysis frequency during the COVID-19 pandemic. BMC Nephrol. 2020; $21: 532$.

13 COVID-19 surveillance reports. The Renal Association Website. https://renal.org/sites/ renal.org/files/ALL_REGIONS_CENTRES covid_report_01072020.pdf.

14 Langley GL, Moen R, Nolan KM, Norman CL, Provost LP. The improvement guide: a practical approach to enhancing organizational performance. 2nd ed. San Francisco: JosseyBass Publishers; 2009.

15 Gray S, Clough T, Mcgee Y, Murphy T, Poulikakos D. Increased risk of COVID-19 in haemodialysis healthcare workers in a tertiary centre in the North West of England. J Hosp Infect. 2020;106(2):390-1.

16 Evaluation of sensitivity and specificity of four commercially available SARS-CoV-2 antibody immunoassays. Public Health England. https://assets.publishing.service.gov. uk/government/uploads/system/uploads/attachment_data/file/898437/Evaluation__of_ sensitivity_and_specificity_of_4_commercially_available_SARS-CoV-2_antibody_immunoassays.pdf.

17 Anthony S, Amalberti R, Berwick DM, Braithwaite J, Lachman P, Vincent CA. COVID-19: patient safety and quality improvement skills to deploy during the surge. Int J Qual Health Care. 2021;33(1):mzaa050.

18 Chan JF, Yuan S, Zhang AJ, Poon VK, Chan CC, Lee AC, et al. Surgical mask partition reduces the risk of noncontact transmission in a golden syrian hamster model for coronavirus disease 2019 (COVID-19). Clin Infect Dis. 2020 Nov 19;71(16):2139-49.

19 COVID-19 Surveillance Reports. The renal association website; 2021. Available from: https: //renal.org/sites/renal.org/files/ALL_REGIONS_CENTRES_covid_report_29072020. pdf.

20 COVID-19 Surveillance Reports. The renal association website; 2021 . Available from: https: //renal.org/sites/renal.org/files/ALL_REGIONS_CENTRES_covid_report_130121_FINAL.pdf.

21 Clarke CL, Prendecki M, Dhutia A, Gan J, Edwards C, Prout V, et al. Longevity of SARS$\mathrm{CoV}-2$ immune responses in hemodialysis patients and protection against reinfection. Kidney Int. 2021 Mar 24;99(6):14700029514777. 